\title{
Estudio de prospectiva del sector cacao al año 2032 como base de programas de capacitación universitaria en el sector agroindustrial. Aplicación del método Delphi
}

\author{
Diana C. Meza-Sepulveda ${ }^{\star}$, Jorge I. Quintero-Saavedra ${ }^{1}$ y Jhon W. Zartha-Sossa ${ }^{2}$, Raul Hernández-Zarta ${ }^{2}$ \\ (1) Facultad de Ciencias Agrarias y Agroindustria, Universidad Tecnológica de Pereira, Carrera 27 \#10-02 Pereira - \\ Risaralda - Colombia (correo-e: dcmeza@utp.edu.co; j.quintero1@utp.edu.co) \\ (2) Facultad de Ingienería Agroindustrial, Universidad Pontificia Bolivariana, Circular $1 N^{\circ} 70-01$, Medellín - Antioquia - \\ Colombia (correo-e: jhonzartha@upb.edu.co; rhernandezz@uqvirtual.edu.co)
}

Recibido Dic. 23, 2019; Aceptado Feb. 18, 2020; Versión final Abr. 20, 2020, Publicado Jun. 2020

\begin{abstract}
Resumen
En este artículo se describen las actividades y resultados del proyecto "Alianza Regional para el Fortalecimiento del Sector Agroindustrial en Risaralda" - Componente Prospectiva, mediante la aplicación del método Delphi a dos rondas, a través de la invitación a grupos de interés del sector con el fin de contribuir a reducir la incertidumbre en la toma de decisiones proponiendo temas, innovaciones y tecnologías que se convertirán en apuesta para el desarrollo de nuevos y/o mejorados programas de capacitación en el sector agroindustrial de Risaralda al 2032, entre los resultados más importantes se priorizaron 42 temas y tecnologías en cuatro agrupadores; los cuales se presentan como insumo para la realización de estrategias de capacitación y transferencia. Como conclusión se resaltan siete estrategias y programas de capacitación sobre formación en buenas prácticas; para lograr certificaciones internacionales, cursos para adopción y transferencia de tecnologías emergentes en nanotecnología en fibras o cascarilla, generación de espacios de ideación y trabajo colaborativo.
\end{abstract}

Palabras clave: prospectiva; método Delphi; grupos de interés; agroindustria; cacao

\section{Prospective study of the cacao sector to the year 2032 as basis of university training programs in the agribusiness sector. Application of Delphi method}

\begin{abstract}
This article presents the activities and results of the project "Regional Alliance for Strengthening the AgroIndustrial Sector in Risaralda" - Prospective Component, based on the application of two rounds of the Delphi method. This was conducted through involvement of stakeholders within the sector to reduce uncertainty in decision-making by creating topics, innovations and technologies that could be leveraged for the development of better or new capacity-building programs in the agro-industrial sector in Risaralda in 2032. Amid the most important results, 42 themes and technologies were prioritized in four groups, of which four groups presented input into capacity strategies building and transference. As a conclusion, the results indicated 6 strategies and new capacity programs for good practices to achieve international certifications, courses on adaptation and transference of emerging technology in nanotechnology in fibers or husks, as well as a the growth of an ideation and co-working.
\end{abstract}




\section{INTRODUCCIÓN}

La demanda mundial de cacao ha crecido, trascendiendo gustos culturales, se expandió en comercialización, industrialización, producción a otros continentes. El consumo mundial alcanzó en 2013 el nivel de 4 millones de toneladas. En 2014 hay déficit de suministro, elevando su precio internacional en 10\%, esta oportunidad de mercados sustenta la inversión económica. Colombia presenta niveles de producción moderadamente bajos pero de altísima calidad (fino y de aroma), está llamado a producir más, manteniendo esa calidad distintiva, a mejorar procesos productivos, hacerlos eficientes y generar cadena productiva que integre en ella hacia adelante a comunidades de economía campesina, en transformación, y comercialización; superar el nivel exportador de productos, integrando a todos los actores generando cultura eficiente e incluyente alrededor del mismo (Ruiz, 2014).

El país dentro de sus políticas de competitividad ha identificado el cacao entre los productos con mayor potencial de producción y exportación a mercados norteamericanos, europeos y asiáticos. El producto cacao está reconocido por los consumidores por la calidad de la taza y su fino aroma, variables que lo cualifican como competitivo en mercados exigentes. (Carvajal, 2015) Ben Martin (1995), describe la prospectiva como "El proceso de investigación que requiere mirar sistemáticamente el futuro de largo plazo en ciencia, tecnología, economía y sociedad, con el objetivo de identificar las áreas de investigación estratégicas y las tecnologías genéricas emergentes que generarán los mayores beneficios económicos y sociales", cuando se habla de prospectiva, se está haciendo referencia a una herramienta que ofrece una ayuda para la aproximación de construcción del futuro (Cepal, 2013).

El método Delphi consiste en una consulta estructurada, anónima y reiterativa a expertos, con el objeto de identificar opciones, obtener consenso y validar resultados (Zartha, et al. 2015). Existen dos tipos de elementos para identificar el futuro, el exploratorio, que busca identificar escenarios futuros, y los estudios normativos, que están dirigidos a priorizar un listado de temas o tecnologías genéricas, con la posibilidad de agregar temas o tecnologías a los inicialmente propuestos, y otras que considere el experto que puedan hacer falta. Este artículo presenta los resultados de un proyecto entre la Universidad Tecnológica de PereiraColombia y el Ministerios de Educación Nacional, relacionado con la alianza regional para el fortalecimiento del sector agroindustrial en Risaralda, en el objetivo de realización de un estudio de prospectiva sobre las necesidades del Departamento de Risaralda - Colombia para su consolidación como territorio de vocación agrícola sostenible, este artículo busca responder la siguiente pregunta ¿Cuáles son las líneas, áreas temáticas, tecnologías, innovaciones y posibles nuevas ofertas de capacitación y/o transferencia que serán representativas para el 2032 en el sector Cacao para Risaralda?

En el contexto latinoamericano en el año 2018 se consolidó la iniciativa de una plataforma multi agencia de cacao para América Latina y el Caribe: Cacao 2030-2050, que permitirá consolidar el potenciamiento y desarrollo de nichos de mercado para los países participantes; fortaleciendo áreas como la producción, productividad, calidad, industria, mercados y regulaciones. Basada en la investigación, el desarrollo tecnológico y el intercambio de conocimientos y experiencias para fortalecer la producción de cacao de calidad y libre de contaminantes en la región (Castro el al., 2018). Alineado a esto en Colombia se realizó en 20182019; un proyecto de USAID/USDA, Cacao para la Paz (CpP), que buscaba fortalecer las instituciones agrícolas clave para el cacao del sector público y privado en Colombia, mediante investigación cooperativa, asistencia técnica, extensión y educación. Que como resultado en su informe examinó en detalle la cadena productiva del cacao en determinadas regiones del país, estudió oportunidades y fortalezas con productores y actores clave, y ofreció enfoques estratégicos para posicionar al sector cacaotero colombiano en el mercado nacional e internacional (Abbott et al., 2017)

Benjamin et al., (2019), plantea que "el sector del cacao colombiano presenta oportunidades específicamente en el contexto del desarrollo post-conflicto. Los esfuerzos recientes para promover el sector se han centrado en la expansión de la producción de cacao y en un menor grado de gestión posterior a la cosecha, el establecimiento de organizaciones de productores y la exploración de nichos de mercado. A pesar de estas intervenciones, el sector todavía sub cumple su potencial. En lugar de centrarse principalmente en la producción de cacao, proponemos una estrategia que clarifique los roles y responsabilidades en el sector para evitar ineficiencias y solapamientos y así mejorar la coordinación y la colaboración entre los actores nacionales y regionales, inversiones en el fortalecimiento de las organizaciones de productores para que se conviertan en empresas rurales viables, la provisión de señales de mercado claras e incentivos para mejorar las mejores prácticas de gestiónl. Después de considerar todas las aportaciones de los interesados y los datos disponibles, creemos que estas intervenciones mejorarán la competitividad y la productividad de la producción de cacao puede ayudar al sector del cacao a vivir a la capacidad de su potencial". 
La agroindustria busca agregarle valor a la producción agropecuaria que se da en Risaralda y la región próxima como es el Eje Cafetero. La buena oferta ambiental y diversos pisos térmicos, ofrecen oportunidades para desarrollar nuevos productos con mayor grado de sofisticación, a partir de la ciencia, la tecnología y la innovación. Cerca de 1.900 hectáreas cultivadas con este grano han hecho que en siete municipios de Risaralda aumente la producción agrícola, representando un crecimiento económico para los pequeños productores. Es importante destacar que al menos 2.000 familias que se dedicaban a cultivar café han migrado a otros sectores como el plátano, el aguacate y, ahora, el cacao. Si bien el cacao se ha convertido en una nueva alternativa para los caficultores risaraldenses, los diferentes gremios de la agricultura de esta región aseguran que se deben fortalecer ambos sectores; por un lado, el café para no poner en riesgo la declaratoria de Paisaje Cultural Cafetero y, además, el cacao para promover nuevas economías en el campo de este departamento (FEDECACAO, 2019).

La prospectiva puede considerarse como la "Disciplina de gestión que permite reflexionar sobre el futuro - lo que podría ser y lo que debería ser -, con miras a orientar la acción humana - individual o colectiva- en el presente, mediante toda una infraestructura conceptual y metodológica aplicable a problemas de diferentes campos y áreas" (Godet, 1997). Diversos estudios que se han realizado en Colombia de prospectiva han tenido como objetivo la identificación de las necesidades futuras de los recursos humanos que surgirá del impacto de los avances tecnológicos y las tendencias organizacionales entre los 5 y 10 años (Zartha et al., 2017), entre los estudios del sector agroindustrial realizados en Colombia en los últimos años se resaltan en empaques biodegradables, sector piscícola, cafés especiales y estudios previos de futuro en cacao (Castellanos et al., 2007).

La producción científica de estudios de caso sobre el método Delphi es muy amplia, en los últimos años se cuenta con estudios como (Munzberg et al., 2017) mencionan el uso del Delphi con la participación de profesionales y no solamente de expertos académicos, además, (Di zio et al., 2017) trabajaron acerca de elementos territoriales o geográficos por lo que utilizaron un Delphi Espacial. Para Castelló y Callejo, el método Delphi consiste en una consulta a un número de expertos de los sectores o temas específicos elegidos, sobre la base de un cuestionario, preparado por paneles o comisiones de expertos, que se responde anónimamente y en dos o más rondas (Castelló et al., 2004). El método Delphi consiste en una consulta estructurada, anónima y reiterativa a expertos, con el objeto de identificar opciones, obtener consenso y validar resultados (Ramírez et al., 2016). Existen dos tipos de elementos para identificar el futuro, el exploratorio que busca identificar escenarios futuros, y los estudios normativos, que están dirigidas a priorizar un listado de temas o tecnologías genéricas, con la posibilidad de agregar a los temas o tecnologías inicialmente propuestas y otras que considere el experto que puedan hacer falta (Steurer, 2011).

El Delphi es uno de los métodos más reconocidos y utilizados a nivel empresarial y académico, y su aplicación busca ayudar a reducir la incertidumbre en la toma de decisiones, y a su vez contribuir como lo explica Godet a "anticipar y actuar a través de la apropiación" (Godet, 1993). El Delphi consiste en la selección de un grupo de expertos a los que se les pregunta su opinión sobre cuestiones referidas a acontecimientos del futuro. (Nazarko et al., 2015). La calidad de los resultados depende del cuidado que se ponga en la elaboración del cuestionario y en la elección de los expertos consultados. (Andersen et al, 2004). Las estimaciones de los expertos se realizan en sucesivas rondas, anónimas, con el fin de conseguir consenso, pero respetando la opinión y autonomía de los participantes (Gnatzy et al., 2011). El método Delphi permite tener juicios grupales después de rondas sucesivas que brindan aspectos, justificaciones más efectivas que si se tuvieran juicios individuales. (Santos et al., 2017). Por otra parte, se hace una retroalimentación a las respuestas en cada ronda, con el fin de proporcionar la información suficiente para la siguiente ronda. Además, las respuestas se analizan con procedimientos estadísticos descriptivos, con el fin de llegar a un consenso (Gomez et al., 2015).

\section{METODOLOGÍA}

Las actividades realizadas durante cada una de las fases del estudio de prospectiva y los temas y decisiones medulares en los talleres realizados, fueron construidas y validadas por docentes e investigadores de la Universidad Tecnológica de Pereira - Colombia, Facultad de Ciencias Agrarias y Agroindustria y posteriormente socializadas. Estas se resumen en la tabla 1 y cada fase se explica con mayor detalle a continuación.

\section{Fase I}

Esta etapa comprendió la revisión de antecedentes, análisis de documentos de vigilancia tecnológica del sector cacao, con el fin de extraer variables que se convirtieron en insumo del árbol temático y tecnológico. La metodología llevada a cabo para las actividades de vigilancia tuvo como base estudios previos como el de (López, 2016) y de otros subsectores agroindustriales como el de derivados piscícolas (Zartha et al., 2017), subproductos de la curtición (Padilla et al., 2018). 
Tabla 1: Metodología identificada por fases

\begin{tabular}{|l|l|l|}
\hline \multicolumn{1}{|c|}{ Fase I } & \multicolumn{1}{|c|}{ Fase II } & \multicolumn{1}{c|}{ Fase III } \\
\hline $\begin{array}{l}\text { Revisión de antecedentes, análisis } \\
\text { de reportes de Vigilancia Tecnológica } \\
\text { del sector cacao }\end{array}$ & $\begin{array}{l}\text { Elaboración cuestionario de la } \\
\text { primera ronda a 100 expertos }\end{array}$ & $\begin{array}{l}\text { Elaboración cuestionario segunda } \\
\text { ronda - Envío de la segunda ronda }\end{array}$ \\
\hline $\begin{array}{l}\text { Construcción perfil de expertos - } \\
\text { Elaboración de la lista de expertos }\end{array}$ & $\begin{array}{l}\text { Análisis estadístico primera ronda } \\
\text { Delphi e informe ejecutivo }\end{array}$ & $\begin{array}{l}\text { Análisis estadístico de la segunda } \\
\text { ronda }\end{array}$ \\
\hline $\begin{array}{l}\text { Construcción del árbol temático y } \\
\text { tecnológico }\end{array}$ & $\begin{array}{l}\text { Propuesta de cursos de formación y } \\
\text { otras estrategias sobre los temas y } \\
\text { tecnologías prioritarias en la segunda } \\
\text { ronda }\end{array}$ \\
\hline
\end{tabular}

También comprendió la construcción del árbol temático y tecnológico, este árbol tecnológico hace referencia al conglomerado de temas resultantes de la vigilancia tecnológica realizada en bases de datos científicas según estudios previos. En esta fase también se realizó la construcción del perfil de expertos y revisión de la lista de expertos, se tuvo en cuenta que existiera un equilibrio en cuanto a expertos de universidades, empresas, estado y entidades de interfaz tales como: Centros de Desarrollo Tecnológico (CDTs), centros de productividad, incubadoras de empresas y parques científicos y tecnológicos entre otros.

\section{Fase II}

En esta fase se llevó a cabo la elaboración del cuestionario para la primera ronda Delphi, se realizó con base en el árbol de temas y tecnologías para cada agrupador, los expertos contestaron la importancia o prioridad de cada tema o tecnología a 2032 en escala likert, la escala Likert es una medición que se utiliza para comprender las opiniones y actitudes de un experto hacia un proyecto, servicio, meta (Question Pro, 2018, citados por Gamboa y Restrepo, 2018), es propio de variables ordinales en el que los valores indican un orden de jerarquía, ha sido usada en estudios de prospectiva para priorizar tecnologías, innovaciones en escalas de que va de 0 -ninguna prioridad a 5 - alta prioridad (Foster, 2015), (Meijering et al., 2016), aunque también pueden tomar valores de 0 a 4,0 a 7 y 0 a 9 , conservando el orden de prioridad desde un mínimo hasta un máximo valor, en esta ronda se solicitó a los participantes anexar comentarios o justificaciones para los temas y tecnologías. Una vez recibida la primera ronda Delphi debidamente diligenciada, se procedió a realizar el análisis estadístico donde se utiliza diferentes técnicas estadísticas tales como: moda, frecuencia modal, porcentaje de consenso, técnicas que han sido referenciadas en estudios previos (Zartha, Halal y Hernández, 2019).

\section{Fase III}

En esta fase se realizó la construcción y envío de la segunda ronda Delphi, la cual buscaba retroalimentar los resultados obtenidos en la primera ronda, debido a que en esta etapa se le solicita al experto que al momento de diligenciar la encuesta que "suba" los temas y tecnología en discusión al grupo de prioritarios y que "baje" aquellos que ve en prioritarios pero que considera que deberían estar en el grupo de discusión. Se aplicaron dos rondas de acuerdo con las tendencias de reducción del número de rondas y a los resultados de estudios previos realizados por (Adini et al., 2017; Durante y Schwartzman, 2017; Varho et al., 2016) y otras investigaciones en agroindustria. Después de recibir la segunda ronda Delphi debidamente diligenciada, se procedió a realizar el análisis estadístico donde se utiliza las técnicas estadísticas descritas para la primera ronda, en esta etapa, se presentan los resultados finales de la aplicación del método Delphi, donde se presentan luego de las 2 rondas Delphi los agrupadores con sus respectivos temas prioritarios, según estudios previos realizados por (Rowe et al., 1999)

\section{RESULTADOS Y DISCUSIÓN}

Los resultados se presentan por cada una de las tres fases, analizando en cada caso el significado y alcance de dichos resultados.

\section{Fase I}

Se presentan los resultados obtenidos en las fases del método Delphi. Para la elaboración de la primera ronda Delphi se presentaron a los expertos un total de 4 agrupadores con 83 temas en Cacao, que fueron incluidos en el formato de primera ronda. Después de que el experto diligenció la información inicial sobre afiliación y nivel de formación, contestó el cuestionario que incluía las variables detalladas en la tabla 2. 
Tabla 2: Variables primera ronda Delphi CACAO

\begin{tabular}{|c|c|}
\hline \multicolumn{2}{|l|}{ Variables genéricas CACAO } \\
\hline Tecnologías transgénicas en semillas & Sombrío transitorio \\
\hline Calidad de grano & Sombrío permanente \\
\hline Semillas certificadas & Injertación \\
\hline $\begin{array}{l}\text { Bioprospección para identificar y caracterizar nuevas } \\
\text { variedades. }\end{array}$ & Manejo - Control de arvenses \\
\hline $\begin{array}{l}\text { Herramientas avanzadas para edición de genes de } \\
\text { productos agrícolas (e.g. CRISPR para desarrollar nuevas } \\
\text { variedades/híbridos). }\end{array}$ & Manejo - Control de plagas \\
\hline Clones de Cacao propagados por métodos asexuales. & Manejo integrado de cultivo \\
\hline $\begin{array}{l}\text { Mejoramiento de las prácticas culturales de acuerdo con } \\
\text { cada cultivo. }\end{array}$ & Manejo sanitario \\
\hline $\begin{array}{l}\text { Adopción de nuevas tecnologías para las prácticas culturales } \\
\text { de acuerdo con cada cultivo. }\end{array}$ & Podas \\
\hline $\begin{array}{l}\text { Difusión de nuevas tecnologías para las prácticas culturales } \\
\text { de acuerdo con cada cultivo. }\end{array}$ & Acceso a asistencia técnica \\
\hline $\begin{array}{l}\text { Macrodifusión (Difusión de una tecnología en una sociedad) } \\
\text { de nuevas tecnologías para las prácticas culturales. }\end{array}$ & Acceso a la información acerca del cultivo \\
\hline $\begin{array}{l}\text { Macrodifusión (Difusión de una tecnología en una } \\
\text { organización determinada) de nuevas tecnologías para las } \\
\text { prácticas culturales. }\end{array}$ & $\begin{array}{l}\text { Tratamientos y preparaciones de suelo que reducen la } \\
\text { absorción de cadmio en el cacao }\end{array}$ \\
\hline Selección de tecnologías para cada tipo de productor. & $\begin{array}{l}\text { Desarrollo de nuevas variedades que reducen los niveles } \\
\text { de cadmio en el cacao }\end{array}$ \\
\hline Resistencia al cambio por parte del productor & Plan de manejo ambiental \\
\hline $\begin{array}{l}\text { Conocer el ciclo de vida de las actuales tecnologías para } \\
\text { cada cultivo. }\end{array}$ & Desarrollo de estándares para el beneficio del grano \\
\hline Heterogeneidad en la densidad de siembra & Normalización del control de calidad del grano \\
\hline Preparación del suelo (limpieza, trazado, ahoyado). & $\begin{array}{l}\text { Propagación de material vegetal mediante Embriogénesis } \\
\text { Somática. }\end{array}$ \\
\hline Análisis de suelo & Uso de Sistemas Agroforestales (SAF) \\
\hline Necesidades químicas y físicas del suelo & Certificación de cultivos orgánicos \\
\hline Desinfestado de hoyos & Sistemas de beneficio eficientes \\
\hline Fertilizado de hoyos & Nuevos métodos de fermentación \\
\hline Trazados de siembra & Buenas practicas agricolas (BPA) \\
\hline Características de la plántula (vigor, edad, tamaño). & Producción orgánica de cacao \\
\hline \multicolumn{2}{|l|}{ Transformación } \\
\hline Colorantes naturales & Producción de nutracéuticos a partir de cacao \\
\hline Saborizantes naturales & Tecnologías de secado emergentes \\
\hline Conservantes naturales & Milkcrumb \\
\hline Conservantes nano-encapsulados & Productos para la industria cosmética \\
\hline Bioconservación vía microorganismos & $\begin{array}{l}\text { Tecnificación del proceso de beneficio del grano de } \\
\text { cacao }\end{array}$ \\
\hline Nanoemulsiones & Sistemas de trazabilidad del producto \\
\hline Obtención de fibras & Acceso a asistencia social \\
\hline Servicio ambiental para captura de carbono & Certificaciones nacionales \\
\hline Cacaocultura inteligente & Manejo de residuos agrícolas \\
\hline $\begin{array}{l}\text { Cacaocultura tradicional para disminuir gases de efecto } \\
\text { invernadero }\end{array}$ & Denominación de origen \\
\hline Polinización del cacao & Transferencia de tecnología \\
\hline Certificaciones internacionales & \\
\hline \multicolumn{2}{|l|}{ Comercialización } \\
\hline Nuevos productos (alimentarios y no alimentarios) & Planificación empresarial \\
\hline Asociatividad & Capacitación empresarial \\
\hline Exportación & $\begin{array}{l}\text { Desarrollo de infraestructura para ayudar al acceso al } \\
\text { mercado }\end{array}$ \\
\hline Importación & $\begin{array}{l}\text { Técnicos capacitados y personal de extensión para } \\
\text { ayudar a los agricultores y procesadores }\end{array}$ \\
\hline Alianza productores/Agroindustria/mercado en fresco & Informes de precios y producción \\
\hline \multicolumn{2}{|l|}{ Fuentes de financiación } \\
\hline \multicolumn{2}{|l|}{ Logística } \\
\hline Vías de acceso & Almacenamiento \\
\hline Transporte & Empaques, embalaje \\
\hline Intermediarios & \\
\hline
\end{tabular}


Ya con el cuestionario de la primera ronda Delphi y la lista de 100 expertos, se procedió a redactar una carta de invitación a participar del estudio de prospectiva. Estos dos archivos fueron diseñados por el equipo monitor y enviados a los expertos en el sector de Cacao de Risaralda, Colombia y otros países. Después de cerrar la fecha de recepción de cuestionarios de primera ronda, se recibieron un total de 17 respuestas en Cacao, sobre las cuales se aplicó un análisis estadístico que incluyó el cálculo de la de moda, la frecuencia modal, el porcentaje de consenso con los temas prioritarios, no prioritarios y en discusión. Con base en lo anterior, se procedió a elaborar el informe ejecutivo de la primera ronda Delphi. Este fue un insumo fundamental para los expertos que contestaron la segunda ronda, ya que una de las características del método Delphi es la retroalimentación para el experto, en número de justificaciones o retroalimentaciones obtenidas en la primera ronda fue de 73.

\section{Fase II}

Se relacionan en la la tabla 3 , el perfil de los 17 expertos que participaron en el estudio se contó con la participación de 11 académicos, 3 empresarios y 3 representantes de instituciones públicas, dentro del grupo se contó con aportes de 2 expertos de Ecuador y 1 de Brasil. Después de los análisis estadísticos realizados de acuerdo con el método mencionado en la metodología, se hallaron, temas prioritarios: 42, temas no prioritarios: 0, temas en discusión: 36, temas No Sabe / No Responde: 5, temas Nuevos: 1.

Tabla 3: Relación de los expertos consultados

\begin{tabular}{|c|c|c|c|c|}
\hline No. & Nombre & Nacionalidad & Afiliación Laboral & Formación Académica \\
\hline 1 & $\begin{array}{l}\text { Carlos Alberto Parra } \\
\text { Salinas }\end{array}$ & Colombiana & Docente/ Universidad de Caldas & $\begin{array}{l}\text { Magíster en Sistemas de } \\
\text { Producción Agropecuaria }\end{array}$ \\
\hline 2 & $\begin{array}{l}\text { Carlos Eduardo Orrego } \\
\text { Alzate }\end{array}$ & Colombiana & $\begin{array}{l}\text { Docente/ Universidad Nacional de } \\
\text { Colombia }\end{array}$ & Doctor en Ciencias-Química \\
\hline 3 & $\begin{array}{l}\text { Diana Carolina Meza } \\
\text { Sepulveda }\end{array}$ & Colombiana & $\begin{array}{l}\text { Docente/ Universidad Tecnológica } \\
\text { de Pereira }\end{array}$ & $\begin{array}{l}\text { Magister en Desarrollo } \\
\text { Agroindustrial }\end{array}$ \\
\hline 4 & Carolina Patiño Giraldo & Colombiana & $\begin{array}{l}\text { Jefe de operaciones - Industrias } \\
\text { Alimenticias Monserrat }\end{array}$ & $\begin{array}{l}\text { Magister en Desarrollo } \\
\text { Agroindustrial }\end{array}$ \\
\hline 5 & $\begin{array}{l}\text { César Augusto Martínez } \\
\text { García }\end{array}$ & Colombiana & $\begin{array}{l}\text { Auxiliar de Investigación - Grupo de } \\
\text { Biodiversidad y Biotecnología UTP }\end{array}$ & Químico Industrial \\
\hline 6 & $\begin{array}{l}\text { Diego Fernando Sierra } \\
\text { Salas }\end{array}$ & Colombiana & Profesional AgroSena & Ingeniero Agroindustrial \\
\hline 7 & Efrain Rodriguez Alzate & Colombiana & Profesional Sena Regional Quindío & Ingeniero Agrónomo \\
\hline 8 & $\begin{array}{l}\text { Jhon Alexander Velez } \\
\text { Montes }\end{array}$ & Colombiano & $\begin{array}{l}\text { Facultad de Ciencias Agrarias y } \\
\text { Agroindustria - UTP }\end{array}$ & $\begin{array}{l}\text { Magister en Desarrollo } \\
\text { Agroindustrial }\end{array}$ \\
\hline 9 & $\begin{array}{l}\text { Juan Antonio Grisales } \\
\text { Adarve }\end{array}$ & Colombiano & Consultor GC Agregando Valor & Ingeniero Industrial \\
\hline 10 & $\begin{array}{l}\text { Juan Manuel Andrade } \\
\text { Navia }\end{array}$ & Colombiano & $\begin{array}{l}\text { Docente/ Corporación Universitaria } \\
\text { Minuto de Dios }\end{array}$ & Candidato a Doctor en Agroindustria \\
\hline 11 & Lina María Suárez Guzmán & Colombiano & $\begin{array}{l}\text { Docente/ Universidad Tecnológica } \\
\text { de Pereira }\end{array}$ & $\begin{array}{l}\text { Magister en Ciencias e Ingeniería de } \\
\text { Alimentos }\end{array}$ \\
\hline 12 & Lucas Penagos Velez & Colombiana & $\begin{array}{l}\text { Jefe Investigación - Nacional de } \\
\text { Chocolates }\end{array}$ & $\begin{array}{l}\text { Doctor en Ciencia y Tecnología de } \\
\text { Alimentos }\end{array}$ \\
\hline 13 & Oswaldo Osorio Mora & Colombiana & $\begin{array}{l}\text { Coordinador del Doctorado en } \\
\text { Ciencias Agrarias Universidad de } \\
\text { Nariño }\end{array}$ & Doctor en Ingeniería de Alimentos \\
\hline 14 & Raúl Hernández Zarta & Colombiana & $\begin{array}{l}\text { Profesional Especializado - } \\
\text { Universidad Nacional de Colombia }\end{array}$ & $\begin{array}{l}\text { Magister en Innovación y } \\
\text { Agronegocios }\end{array}$ \\
\hline 15 & Sergio Pino Perala & Ecuatoriana & Docente / Universidad de Guayaquil & $\begin{array}{l}\text { Doctor en Ciencias Ambientales y } \\
\text { Economía Agraria }\end{array}$ \\
\hline 16 & $\begin{array}{l}\text { Katianny Gomes Santana } \\
\text { Estival }\end{array}$ & Brasilera & $\begin{array}{l}\text { Docente/ Universidad Estatal de } \\
\text { Santa Cruz }\end{array}$ & $\begin{array}{l}\text { Doctora en Ciencias Sociales en } \\
\text { Desarrollo, Agricultura y sociedad }\end{array}$ \\
\hline 17 & Ignacio Antonio Sotomayor & Ecuatoriana & $\begin{array}{l}\text { Investigador Agropecuario - EET } \\
\text { Pichilingue Iniap }\end{array}$ & $\begin{array}{l}\text { Magister en Mejora Genética } \\
\text { Vegetal }\end{array}$ \\
\hline
\end{tabular}


La selección de los agrupadores propuestos; se basó en la definición de "Ingeniería Agroindustrial" la cual comprende la producción, transformación y comercialización de materias primas provenientes de los sectores agrícola, pecuario, forestal y pesquero, para un uso y necesidades de un consumidor total o intermedio, (FAO, 2013), por lo que todos los temas del árbol temático/tecnológico del subsector Cacao se clasificaron dentro de esos agrupadores. En la figura 1, se muestran los resultados obtenidos en los temas priorizados por cada agrupador $60 \%$ en cacao, $19 \%$ transformación, $14 \%$ comercialización y $7 \%$ en logística.

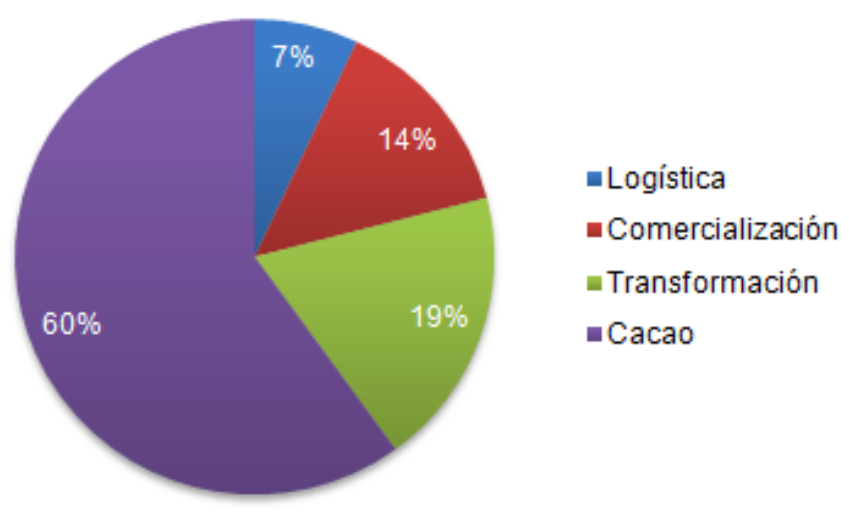

Fig. 1: Temas prioritarios identificados por agrupador

Fase III:

La construcción del cuestionario de segunda ronda Delphi, se realizó con base en el resultado de la primera ronda. En las instrucciones se presentó una explicación de cómo contestar cada agrupador (variables genéricas de cacao, transformación, comercialización y logística), ya que los cambios que el experto realizó eran diferentes para cada agrupador. Básicamente el experto tuvo que leer los aspectos relevantes que en la primera ronda se consolidaron para cada tema de cada agrupador. Con base en la información suministrada, el experto pudo establecer sí un ítem que estaba en prioritario, debería pasar a discusión, y de los temas en discusión, debería pasar a prioritario. Después de cerrar la recepción de respuestas, se procedió a realizar el análisis estadístico donde se utilizaron las técnicas estadísticas descritas para la primera ronda, además, se calculó la frecuencia modal 2 (Fm2) la cual contiene la frecuencia modal 1(Fm1) más el número de expertos que "entran" el tema o tecnología en la ronda dos (pero que en la ronda 1 no lo habían calificado como prioritario) menos los expertos que "sacan" el tema o tecnología en la ronda dos (pero que en la ronda uno lo habían calificado como prioritario), permitiendo de esta forma obtener los temas prioritarios. En la tabla 4, se observan los resultados obtenidos del análisis de la segunda ronda Delphi en el sector Cacao en comparación con la primera ronda Delphi.

De acuerdo con la tabla anterior se obtuvieron 42 temas y tecnologías prioritarias en las dos rondas, 19 de ellos cumplieron con el requisito de ser prioritarios en ambas rondas. En el grupo 1 de variables genéricas sobre cacao 11 temas y tecnologías fueron prioritarios en las dos rondas, mientras que 12 que no fueron prioritarios en la primera ronda, si entraron en la categoría en la segunda, los temas y tecnologías que cumplieron esta característica fueron: semillas certificadas, bioprospección para identificar y caracterizar nuevas variedades, herramientas avanzadas para edición de genes de productos agrícolas (e.g. CRISPR para desarrollar nuevas variedades/híbridos), microdifusión (Difusión de una tecnología en una organización determinada) de nuevas tecnologías para las prácticas culturales, resistencia al cambio por parte del productor, conocer el ciclo de vida de las actuales tecnologías para cada cultivo, necesidades químicas y físicas del suelo, desinfestado de hoyos, acceso a la información acerca del cultivo, uso de Sistemas Agroforestales (SAF), sistemas de beneficio eficientes y producción orgánica de cacao.

En el grupo 2 de transformación 8 temas y tecnologías fueron prioritarias en la primera ronda, mientras que 6 no fueron prioritarios en la primera ronda, si entraron en la categoría de la segunda ronda los temas y tecnologías, nanoemulsiones, servicio ambiental para captura de carbono, cacaocultura inteligente, certificaciones internacionales, tecnologías de secado emergentes y manejo de residuos agrícolas. En el grupo 3 de comercialización 6 temas y tecnologías fueron prioritarias en la primera ronda, mientras que 3 no fueron prioritarios en la primera ronda pero si entraron como prioritarios en la segunda, los temas fueron fuentes de financiación, planificación empresarial e informes de precios y producción. En el grupo 4 de logística 3 temas y tecnologías fueron prioritarios en la primera ronda, mientras que 2 no fueron prioritarios en la primera ronda, pero si entraron como prioritarios en la segunda ronda, los temas fueron intermediarios y almacenamiento. 
Tabla 4: Resumen de temas y tecnologías prioritarios en las dos rondas Delphi.

\begin{tabular}{|c|c|c|}
\hline & $1^{\mathrm{a}}$ ronda & $2^{-\mathrm{a}}$ ronda \\
\hline Calidad de grano & Prioritario & Prioritario \\
\hline Semillas certificadas & Discusión & Prioritario \\
\hline Bioprospección para identificar y caracterizar nuevas variedades & Discusión & Prioritario \\
\hline Herramientas avanzadas para edición de genes de productos agrícolas & Discusión & Prioritario \\
\hline Clones de Cacao propagados por métodos asexuales & Prioritario & Discusión \\
\hline Mejoramiento de las prácticas culturales de acuerdo a cada cultivo & Prioritario & Prioritario \\
\hline Adopción de nuevas tecnologías para las prácticas culturales de acuerdo a cada cultivo & Prioritario & Prioritario \\
\hline Difusión de nuevas tecnologías para las prácticas culturales de acuerdo a cada cultivo & Prioritario & Prioritario \\
\hline Macrodifusión de nuevas tecnologías para las prácticas culturales & Prioritario & Discusión \\
\hline Microdifusión de nuevas tecnologías para las prácticas culturales & Discusión & Prioritario \\
\hline Selección de tecnologías para cada tipo de productor & Prioritario & Discusión \\
\hline Resistencia al cambio por parte del productor & Discusión & Prioritario \\
\hline Conocer el ciclo de vida de las actuales tecnologías para cada cultivo & Discusión & Prioritario \\
\hline Heterogeneidad en la densidad de siembra & Prioritario & Discusión \\
\hline Preparación del suelo (limpieza, trazado, ahoyado) & Prioritario & Discusión \\
\hline Análisis de suelo & Prioritario & Discusión \\
\hline Necesidades químicas y físicas del suelo & Discusión & Prioritario \\
\hline Desinfestado de hoyos & Discusión & Prioritario \\
\hline Fertilizado de hoyos & Prioritario & Discusión \\
\hline Trazados de siembra & Prioritario & Discusión \\
\hline Injertación & Prioritario & Prioritario \\
\hline Manejo - Control de plagas & Prioritario & Discusión \\
\hline Manejo integrado de cultivo & Prioritario & Prioritario \\
\hline Manejo sanitario & Prioritario & Discusión \\
\hline Podas & Prioritario & Discusión \\
\hline Acceso a asistencia técnica & Prioritario & Prioritario \\
\hline Acceso a la información acerca del cultivo & Discusión & Prioritario \\
\hline Tratamientos y preparaciones de suelo que reducen la absorción de cadmio en el cacao & Prioritario & Prioritario \\
\hline Desarrollo de nuevas variedades que reducen los niveles de cadmio en el cacao & Prioritario & Prioritario \\
\hline Plan de manejo ambiental & Prioritario & Prioritario \\
\hline Desarrollo de estándares para el beneficio del grano & Prioritario & Prioritario \\
\hline Normalización del control de calidad del grano & Prioritario & Discusión \\
\hline Uso de Sistemas Agroforestales (SAF) & Discusión & Prioritario \\
\hline Sistemas de beneficio eficientes & Discusión & Prioritario \\
\hline Nuevos métodos de fermentación & Prioritario & Prioritario \\
\hline Buenas prácticas agrícolas (BPA) & Prioritario & Prioritario \\
\hline Producción orgánica de cacao & Discusión & Prioritario \\
\hline Nanoemulsiones & Discusión & Prioritario \\
\hline Servicio ambiental para captura de carbono & Discusión & Prioritario \\
\hline Cacaocultura inteligente & Discusión & Prioritario \\
\hline Cacaocultura tradicional para disminuir gases de efecto invernadero & Prioritario & Discusión \\
\hline Polinización del cacao & Prioritario & Discusión \\
\hline Certificaciones internacionales & Discusión & Prioritario \\
\hline Transferencia de tecnología & Prioritario & Prioritario \\
\hline Producción de nutracéuticos a partir de cacao & Prioritario & Prioritario \\
\hline Tecnologías de secado emergentes & Discusión & Prioritario \\
\hline Productos para la industria cosmética & Prioritario & Discusión \\
\hline Tecnificación del proceso de beneficio del grano de cacao & Prioritario & Prioritario \\
\hline Sistemas de trazabilidad del producto & Prioritario & Prioritario \\
\hline Manejo de residuos agrícolas & Discusión & Prioritario \\
\hline Denominación de origen & Prioritario & Prioritario \\
\hline Nuevos productos (alimentarios y no alimentarios) & Prioritario & Discusión \\
\hline Asociatividad & Prioritario & Prioritario \\
\hline Exportación & Prioritario & Discusión \\
\hline Alianza productores/Agroindustria/mercado en fresco & Prioritario & Discusión \\
\hline Fuentes de financiación & Discusión & Prioritario \\
\hline Planificación empresarial & Discusión & Prioritario \\
\hline Desarrollo de infraestructura para ayudar al acceso al mercado & Prioritario & Discusión \\
\hline Técnicos capacitados y personal de extensión para ayudar a los agricultores & Prioritario & Discusión \\
\hline Informes de precios y producción & Discusión & Prioritario \\
\hline Vías de acceso & Prioritario & Discusión \\
\hline Transporte & Prioritario & Discusión \\
\hline Intermediarios & Discusión & Prioritario \\
\hline Almacenamiento & Discusión & Prioritario \\
\hline Empaques, embalaje & Prioritario & Discusión \\
\hline Poscosecha & No incluido & Prioritario \\
\hline
\end{tabular}


En la tabla 5 se observa los nuevos temas prioritarios de la 2da ronda Delphi. Los temas y tecnologías presentados en la tabla anterior cumplieron en la primera ronda con el criterio de moda alta 4 o 5 y consenso alto, es decir, por encima del promedio de su grupo, además, en la segunda ronda permanecieron con consenso alto, lo que significa que los grupos de interés participantes no cambiaron sustancialmente de opinión sobre su importancia o prioridad al 2032. Estos temas y tecnologías se constituyen en el primer insumo para los cursos de capacitación y otras estrategias que desde las universidades relacionadas con programas de agroindustria pueden ofrecer a los productores, gremios, empresarios y otros grupos de interés relacionados con la cadena del cacao. Los temas y tecnologías prioritarios en las dos rondas se complementan con aquellos que suben como prioritarios en la segunda ronda, aunque en la primera hayan sido temas en discusión, esto quiere decir que la primera ronda del estudio Delphi su consenso no estuvo por encima del promedio de su grupo, pero en la segunda ronda varios expertos participantes lo incluyeron como prioritario fruto de la retroalimentación que se hace con los comentarios de todos los encuestados y que son insumo de la segunda encuesta. Un análisis final debe tener en cuenta los temas que no eran parte del árbol de temas y tecnologías de la primera ronda, pero que los expertos proponen como un tema nuevo; este fue el caso del tema de "poscosecha" el cual fue propuesto por los expertos como prioritario en la segunda ronda.

Tabla 5: Temas prioritarios en las 2 rondas Delphi

\begin{tabular}{|l|}
\hline Calidad de grano \\
\hline Mejoramiento de las prácticas culturales de acuerdo a cada cultivo \\
\hline Adopción de nuevas tecnologías para las prácticas culturales de acuerdo a cada cultivo \\
\hline Difusión de nuevas tecnologías para las prácticas culturales de acuerdo a cada cultivo \\
\hline Injertación \\
\hline Manejo integrado de cultivo \\
\hline Acceso a asistencia técnica \\
\hline Tratamientos y preparaciones de suelo que reducen la absorción de cadmio en el cacao \\
\hline Desarrollo de nuevas variedades que reducen los niveles de cadmio en el cacao \\
\hline Plan de manejo ambiental \\
\hline Desarrollo de estándares para el beneficio del grano \\
\hline Nuevos métodos de fermentación \\
\hline Buenas prácticas agrícolas (BPA) \\
\hline Transferencia de tecnología \\
\hline Producción de nutracéuticos a partir de cacao \\
\hline Tecnificación del proceso de beneficio del grano de cacao \\
\hline Sistemas de trazabilidad del producto \\
\hline Denominación de origen \\
\hline Asociatividad \\
\hline
\end{tabular}

De acuerdo con los resultados anteriores se recomienda construir de manera colectiva; estrategias, proyectos y programas de capacitación desde las universidades e instituciones de educación relacionadas con la agroindustria en Risaralda, en especial los temas que fueron seleccionados como prioritarios en la primera y segunda ronda, sin embargo, un insumo para estas estrategias y nuevos programas de capacitación se detalla en la siguientes siete etapas: 1) formación en estrategias de buenas prácticas para lograr certificaciones internacionales; 2) cursos para adopción y transferencia de tecnologías emergentes (nanotecnología en fibras o cascarilla); 3) políticas para el fortalecimiento de transformación que beneficien al sector productor; 4) generación de espacios de ideación y trabajo colaborativo (a través de campamentos de entrenamiento y maratones de emprendimiento) en los municipios alrededor de los temas/tecnologías/innovaciones prioritarias, de tal forma que se puedan proponer soluciones o nuevos emprendimientos - empresa emergentes que puedan constituirse en nuevos actores del sistema regional de innovación; 5) co-desarrollar convocatorias para que los grupos de interés del sector de Cacao pueda llevar a cabo iniciativas y proyectos que permitan lograr los indicadores propuestos; 6) desarrollar espacios o mecanismos de política pública (alianzas público privadas, asignación de presupuestos) que logren el cumplimiento de los indicadores; 7) generación de proyectos concretos de I+D+i, cursos de extensión, programas de formación continua y de transferencia en los temas/tecnologías/innovaciones ganadoras en las dos rondas Delphi y los nuevos temas prioritarios de la 2da ronda Delphi.

\section{DISCUSIÓN FINAL}

De los 42 temas y tecnologías prioritarios en la segunda ronda, sólo 19 cumplieron con el requisito de prioritario en ambas rondas, estos temas giran sobre calidad de grano, mejoramiento de las prácticas culturales de acuerdo a cada cultivo, adopción de nuevas tecnologías para las prácticas culturales, difusión 
de nuevas tecnologías para las prácticas culturales, injertación, manejo integrado de cultivo, tratamientos y preparaciones de suelo que reducen la absorción de cadmio, desarrollo de nuevas variedades que reducen los niveles de cadmio, plan de manejo ambiental, desarrollo de estándares para el beneficio del grano, nuevos métodos de fermentación, Buenas Prácticas Agrícolas (BPA), transferencia de tecnología, producción de nutracéuticos, tecnificación del proceso de beneficio del grano, sistemas de trazabilidad del producto, denominación de origen, asociatividad.

Los 2 temas prioritarios con mayor porcentaje de consenso en cada uno de los cuatro agrupadores fueron: Manejo integrado de cultivo, Calidad de grano, Transferencia de tecnología, Producción de nutracéuticos a partir de cacao, Asociatividad, Nuevos productos (alimentarios y no alimentarios), Empaques y Embalajes y Transporte, estos temas se convierten en un primer foco para la generación de estrategias y cursos de capacitación para los actores relacionados con el subsector de cacao en Risaralda. La mayor concentración de temas prioritarios en la segunda ronda se identificaron en los agrupadores "variables genéricas de cacao" y "transformación" con el 85\% de los temas y tecnologías prioritarios, en los agrupadores "comercialización" y "logística" los expertos solo priorizaron 6 temas, esto demuestra el interés o foco de los expertos encuestados sobre los temas de producción y transformación, de hecho el agrupador "logística" no cuenta con temas prioritarios en las dos rondas, y el agrupador "comercialización" sólo aportó uno, el de asociatividad.

Los resultados del estudio son importantes para los empresarios y en general para todos los agentes de los sistemas de innovación del sector de Cacao, tanto para Risaralda como para otras regiones del País que comparten las mismas características, y pueden convertirse en insumos para la elaboración de políticas públicas relacionadas con la cadena del Cacao, estas políticas se podrían constituir en insumos para promover buenas prácticas en gestión de I+D+i y gestión tecnológica en aspectos como compra pública innovadora, instrumentos para incentivar demanda de innovaciones, promoción de cultura innovadora, espacios para el diálogo de políticas en las áreas de acción del estado en la economía y formas de colaboración universidad - empresa. Los resultados del método Delphi se convierten en apuestas directas dentro de la cadena de la l+D+i y de posibles actividades de formación desde las universidades, estos temas y tecnologías trascienden a la región específica donde se realizó el estudio y se convierten en insumos para actores relacionados con el sistema sectorial de ciencia, tecnología e innovación del subsector específico del cacao.

Para la Facultad de Ciencias Agrarias y Agroindustria de la Universidad Tecnológica De Pereira y en general para el sector académico; este articulo proporciona información valiosa, para la consolidación de líneas de investigación y para la creación o actualización de programas de pregrado y posgrado, relacionados con la agroindustria; encaminados a mejorar la calidad y trazabilidad de la materia prima, la caracterización de los subproductos del cacao y la generación de nuevos procesos / productos con alto valor agregado; haciendo enfasis en la sostenibilidad, el encadenamiento productivo y la innovacion.

\section{CONCLUSIONES}

De acuerdo al trabajo presentado y a los resultados obtenidos, se pueden plantear las siguientes conclusiones principales: 1) los pequeños agricultores, comercializadores y funcionarios del gobierno encontrarán en los temas prioritarios resultantes de los agrupadores, una lista de temas y tecnologías que pueden convertirse en foco de próximos proyectos conjuntos dentro de la cadena $1+D+i$, estas nuevas iniciativas podrán analizarse en talleres conjuntos para que sirvan como iniciativas para proyectos de corto, mediano y largo plazo en las regiones; 2) los resultados del estudio son importantes para los empresarios y en general para todos los agentes de los sistemas de innovación del sector de Cacao tanto para Risaralda, como para otras regiones de Países que comparten las mismas características, y pueden convertirse en insumos para la elaboración de políticas públicas relacionadas con la cadena del Cacao.

\section{AGRADECIMIENTOS}

A la Universidad Tecnológica de Pereira, al Ministerio de Educación Nacional y a la Facultad de Ciencias agrarias y Agroindustria, quienes financiaron esta investigación mediante el Convenio Interadministrativo número 0898 de 2018 suscrito entre el Ministerio de Educación Nacional y la Universidad Tecnológica de Pereira.

\section{REFERENCIAS}

Abbott, P.C., Benjamin, T., Burniske, G., Croft, M., Análisis de la Cadena Productiva del Cacao en Colombia, https//doi.org/10.13140/RG.2.2.10934.14400, Technical Report, Universidad de Purdue (2017) 
Adini, B., Cohen, O., Eide, A.W., Nilsson, S., Aharonson-Daniel, L. y Herrera, I.A, Striving to be resilient: What concepts, approaches and practices should be incorporated in resilience management guidelines? https://doi.org/10.1016/j.techfore.2017.01.020, Technological Forecasting and Social Change., 121, 39-49 (2017)

Andersen, P.D., Jørgensen, B.H., Lading, L., y Rasmussen, B., Sensor foresight-technology and market. https://doi.org/10.1016/S0166-4972(02)00072-X, Technovation, 24, 311-320 (2004)

Benjamin, T., Wilcox, M.D., Burniske, G., y Colleen, K., Cacao for Peace (CFP), Universidad de Purdue (2019)

Carvajal, J.C., Perfil tecnológico del comercio rural del cacao en el departamento Norte de Santander. https://doi.org/10.15649/2346030X.514, Revista de Investigación, 3(2), 37 - 46 (2015)

Castellanos, O.F., Torres, L.M., y otros tres autores, Agenda prospectiva de investigación y desarrollo tecnológico para la cadena productiva de cacao-chocolate en Colombia, Universidad Nacional de Colombia, Bogotá, Colombia (2007)

Castelló, M. y Callejo, J., La prospectiva tecnológica y sus métodos, Dirección nacional de planificación y evaluación, Nicaragua (2004)

Castro, L., Gómez, R., y Vignati, F., Iniciativa Latinoamericana del Cacao: Boletín No. 4, Caracas, http://scioteca.caf.com/handle/123456789/1347, (2018)

CEPAL., Prospectiva y desarrollo: El clima de la igualdad en América Latina y el Caribe a 2020. Comisión Económica para América Latina y el Caribe, Santiago de Chile, Chile (2013)

Di Zio, S., Castillo Rosas, J.D., y Lamelza, L., Real Time Spatial Delphi: Fast convergence of experts' opinions on the territory. https://doi.org/10.1016/j.techfore.2016.09.029, Technological Forecasting and Social Change, 115, 143-154 (2017)

Durante, E., y Schwartzman, G., Un novedoso proceso de consulta a los docentes para la definición del perfil de egresado en una carrera de medicina, https://doi.org/10.1016/j.edumed.2016.04.002, Educación Médica, 18(1), 56-60 (2017)

Fedecacao. Boletín de prensa. Federación Nacional de Cacaoteros, Bogotá, Colombia (2019)

Foster, B., Technology foresight for sustainable production in the German automotive supplier industry. https://doi.org/10.1016/j.techfore.2014.09.010, Technological Forecasting and Social Change, 92, 237 - 248, (2015)

Gamboa, A., Restrepo, D., Centro de Servicio de Conocimiento como estrategia para el mejoramiento de la productividad en los proyectos del sector de Ingeniería y Construcción (E\&C) en Colombia. Tesis de Magister. Escuela de Administración. Universidad EAFIT, Medellín, Colombia (2018)

Gnatzy, T., Warth, J., von der Gracht, H., y Darkow, I. L., Validating an innovative real-time Delphi approach-A methodological comparison between real-time and conventional Delphi studies, https://doi.org/10.1016/j.techfore.2011.04.006, Technological Forecasting and Social Change, 78(9), 1681-1694 (2011)

Godet, M., De la anticipación a la acción: Manual de prospectiva y estrategia. 1ra edición. ISBN: 8426709249. Marcombo, S.A, 378, (1993)

Godet, M. Manuel de prospective stratégique, tome 2: L'art et la méthode. Paris (1997)

Gomez, M. A., Camarero, A., y González, N., Use of the Delphi method to determine the constraints that affect the future size of large container ships. https://doi.org/10.1080/03088839.2013.870358, Maritime Policy \& Management, 42(3), 263$277(2015)$

López, A., Méndez, D., Paz, A., y Arboleda, H., Desarrollo e Instrumentación de un Proceso de Vigilancia Tecnológica basado en Protocolos de Revisión Sistemática de la Literatura, http://dx.doi.org/10.4067/S0718-07642016000400017, Información Tecnológica. 27(4), 155-164, (2016)

Martin, B., Foresight in Science and technology. https://doi.org/10.1080/09537329508524202, Technology Analysis \& Strategic Management, 7(2), 139-178 (1995)

Meijering, J.V., y Tobi, H., The effect of controlled opinion feedback on Delphi features: mixed messages from a real-world Delphi experiment. https://doi.org/10.1016/j.techfore.2015.11.008, Technological Forecasting and Social Change, 103, $166-173,(2016)$

Münzberg, T., Wiens, M., y Schultmann, F., A spatial-temporal vulnerability assessment to support the building of community resilience against power outage impacts. https://doi.org/10.1016/j.techfore.2016.11.027, Technological Forecasting and Social Change, 121, 99-118 (2017)

Nazarko, J., Radziszewski, P., y otros once autores, Foresight Study of Road Pavement Technologies. https://doi.org/10.1016/j.proeng.2015.10.016, Procedia Engineering, 122, 129-136 (2015)

Organización de las Naciones Unidas para la Alimentación y la Agricultura FAO., Agroindustrias para el desarrollo, Roma (2013)

Padilla, J., Zartha, J., Álvarez, V., y Orozco, G., Vigilancia Tecnológica para la Identificación de Innovaciones en Subproductos de la Curtición, http://dx.doi.org/10.4067/S0718-07642018000400127, Información Tecnológica, 29(4), 127142, (2018) 
Ramírez, C., Zartha, J., Arango, B., y Orozco, G., Prospectiva 2025 de la Carrera de Ingeniería Química en algunos Países pertenecientes a la Organización de Estados Americanos (OEA). http://dx.doi.org/10.4067/S0718-50062016000600012, Formación Universitaria, 9(6), 127-138 (2016)

Rowe, G., Wright, G. The Delphi technique as a forecasting tool: issues and analysis. https://doi.org/10.1016/S01692070(99)00018-7, International Journal of Forecasting, 15(4), 353-375 (1999)

Ruiz, J.A. Cacao y su aporte al desarrollo colombiano. Ensayo. Facultad de Ciencias Económicas Universidad Militar Nueva Granada, Bogotá, Colombia, https://repository.unimilitar.edu.co/bitstream/handle/10654/13292/ENSAYO\%20Cacao\%20y\%20su\%20aporte\%20al\%20 desarrollo\%20colombiano\%20\%281\%29.pdf?sequence=1\&isAllowed=y (2017)

Santos, C., Araújo, M., Correia, N. A methodology for the identification of strategic technological competences: An application in the sheet metal equipment industry. https://doi.org/10.1016/j.futures.2017.05.002, Futures, 90, 31-45 (2017)

Steurer, J., The Delphi method: An efficient procedure to generate knowledge. https://doi.org/ 10.1007/s00256-011-1145z Skeletal Radiology, 40(8), 959-961 (2011)

Varho, V., Rikkonen, P., y Rasi, S., Futures of distributed small-scale renewable energy inFinland - a Delphi study of the opportunities and obstacles up to 2025. https://doi.org/10.1016/j.techfore.2015.12.001, Technological Forecasting and Social Change, 104, 30-37 (2016)

Zartha, J., Villada, H., y otros ocho autores, Aplication of Delphi Method in a foresight study on biodegradable packaging up to 2032, http://doi.org/ 10.13140/RG.2.1.2288.1123, Espacios. 36(1), 3 (2015)

Zartha, J., Montes, J., Toro, I. H., Delphi Method In Technological Foresight Studies: An approach to calculating the number of experts and the application of the competence coefficient "K" expert. http://dx.doi.org/10.18684/BSAA(15)105-115, Biotecnología en el Sector Agropecuario y Agroindustrial, 15(1), 105-115 (2017)

Zartha, J., Zuluaga, D., Palacio, J., y Montes, J. (2017). Ciclo de Vida de Tecnologías y Curvas en S Aplicadas en Subproductos de la Agroindustria Piscícola, http://dx.doi.org/10.4067/S0718-07642017000200012, Información Tecnológica, 28(2), 105-114, (2017)

Zartha, J.W., Álvarez, V. T., Oviedo, J. C., y Orozco, G. L., Propuesta de Árbol Temático y Tecnológico sobre la Ingeniería Agroindustrial como Herramienta para un Estudio de Prospectiva. http://dx.doi.org/10.4067/S0718-50062019000400013, Formación universitaria, 12(4), 13-26 (2019) 